\title{
L’Église de Sherbrooke et les Trappistes (1880-1948)
}

\section{Jean-Guy Lavallée}

Volume 41, 1974

URI : https://id.erudit.org/iderudit/1007239ar

DOI : https://doi.org/10.7202/1007239ar

Aller au sommaire du numéro

Éditeur(s)

Les Éditions Historia Ecclesiæ Catholicæ Canadensis Inc.

ISSN

0318-6172 (imprimé)

1927-7067 (numérique)

Découvrir la revue

Citer cet article

Lavallée, J.-G. (1974). L’Église de Sherbrooke et les Trappistes (1880-1948).

Sessions d'étude - Société canadienne d'histoire de l'Église catholique, 41, 9-24.

https://doi.org/10.7202/1007239ar

Tous droits réservés @ Les Éditions Historia Ecclesiæ Catholicæ Canadensis Inc., 1975
Ce document est protégé par la loi sur le droit d'auteur. L'utilisation des services d'Érudit (y compris la reproduction) est assujettie à sa politique d'utilisation que vous pouvez consulter en ligne.

https://apropos.erudit.org/fr/usagers/politique-dutilisation/ 


\section{L'Église de Sherbrooke et les Trappistes (1880-1948) ${ }^{1}$}

L'histoire locale et régionale, que le monde savant qualifie de « petite histoire », rejoint la « grande histoire », celle des pays et des institutions. Il n'y a, en fait, ni petite ni grande histoire; il n'y a que l'histoire tout court. C'est ainsi que les études d'histoire locale ou régionale - les monographies, si l'on veut - permettent souvent d'éclairer, de nuancer, d'appuyer, voire de corriger les grandes synthèses. L'histoire des relations entre l'Église de Sherbrooke et l'ordre des Cisterciens ou Trappistes ne s'étudie pas sans que soient touchées les grandes crises d'anticléricalisme et de laïcisme en France au $\mathrm{XIX}^{\mathrm{e}}$ et au $\mathrm{XX}^{\mathrm{e}}$ siècle, l'histoire interne de l'Ordre et certains problèmes beaucoup plus vastes de l'histoire canadienne : l'émigration des Canadiens français vers les États-Unis, principalement en Nouvelle-Angleterre; leur rapatriement dans les limites de la province de Québec; la colonisation de certaines régions moins développées de cette province, comme le Lac Saint-Jean et les Cantons de l'Est, pour n'en citer que deux.

Il n'y eut qu'une véritable tentative d'établissement des Cisterciens ou Trappistes dans le diocèse de Sherbrooke. C'est celle de La Patrie, entre 1880 et 1882, et cette tentative aboutit à un échec. Cependant, à deux reprises par la suite, des Cisterciens ou Trappistes sollicitèrent de l'évêque de Sherbrooke la permission de s'établir dans les limites de son diocèse, une fois en 1903 et une autre fois en 1914. Enfin, en 1944 et en $1948, M^{\text {gr }}$ Desranleau invitait à son tour les Trappistes d'Oka à s'établir dans son diocèse, mais ces derniers ne purent se rendre au désir de l'évêque. Les Trappistes ne s'établirent pas à Sherbrooke, mais le carton Cisterciens-Trappistes, conservé aux archives de l'archevêché de Sherbrooke, montre que plusieurs fidèles, clercs et laïcs du diocèse - des laïcs surtout demandèrent, par l'intermédiaire du maître des novices d'Oka, de

1 Nous tenons à remercier M. le chanoine Provençal, Chancelier, de nous avoir ouvert toutes grandes les portes des archives de l'archevêché de Sherbrooke (AAS) ainsi que M. l'abbé Blais, vice-chancelier, et mademoiselle la secrétaire de la chancellerie pour leur grande serviabilité. Tous trois ont grandement facilité nos recherches. 
celui de Mistassini ou d'autres monastères, des lettres testimoniales afin d'être admis comme novices dans l'Ordre de saint Bernard, au nombre des fils de Rancé. S'il n'y eut jamais de monastère trappiste permanent dans les limites de l'Église de Sherbrooke, il y eut donc des diocésains de cette Église qui firent partie de l'ordre cistercien ou trappiste. Ainsi s'établissait entre une Église locale et un ordre religieux un type de relation qui échappe à l'historien parce qu'il se situe au-delà des seules contingences que ce dernier est à même de saisir.

C'est Pie VII qui, au moment de la Restauration, avec l'aide du cardinal Consalvi, avait travaillé à rétablir l'Ordre de saint Bernard. Mais l'unité n'était pas facile à réaliser. Au milieu du $\mathrm{XIX}^{\mathrm{e}}$ siècle (1847), il y avait la Commune observance et deux branches de l'Etroite observance : l'ancienne réforme, fidèle à Rancé - Sept-Fons en tête - et la nouvelle réforme soucieuse d'un retour aux sources que sont la règle de saint Benoît et les écrits de saint Bernard - Port du Salut en tête - En 1869, Pie IX réalisa une certaine unité entre la Commune et l'Étroite observance, mais elle ne dura pas. En 1891 et 1892, Léon XIII parvint à faire l'unité de l'Etroite observance sous le titre de Ordre des Cisterciens réformés de sainte Marie de la Trappe. L'Etroite observance continue d'être connue sous son nom populaire de Trappistes. Ceux qui vinrent au Canada avant et après 1891 étaient de l'Étroite observance. C'est pourquoi nous parlerons de Trappistes ${ }^{2}$.

En France, l'offensive contre le «cléricalisme » et en faveur de la laïcité prend un caractère d'acharnement entre 1879 et 1886 et ensuite entre 1898 et $1907^{3}$, pour connaître une nouvelle ferveur un peu avant la guerre de $1914^{4}$. Au cours des trois phases de cette crise des relations Eglise-Etat en France, les congrégations sont un sujet de prédilection pour les radicaux et même pour certains modérés. Si, à première vue, cette crise - car il y a crise - semble plutôt toucher les congrégations enseignantes, elle englobe, beaucoup plus qu'on a voulu le laisser voir, toutes les congrégations, enseignantes ou non. C'est ainsi qu'au cours de ces trois phases d'une

2 Dantel-Rops, Saint Bernard et ses fils, Tours, Mame, 1962, pp. 146-152.

3 A. Latreille, E. Delaruelle, J.-R. Palanque et R. Rémond, Histoire du catholicisme en France, III : La période contemporaine, Paris, Éditions Spes, 1962, p. 419.

4 Ibidem, p. 547. 
même crise, dominées par la «mystique anticléricale révolutionnaire », les Trappistes ont raison de tenter de s'établir ailleurs ou, tout au moins, d'y songer. Ces Trappistes cherchent asile dans des régions catholiques où la terre, leur gagne-pain traditionnel, est suffisamment disponible pour assurer leur survie. Ces moments de crise en France coïncident avec une situation problématique dans la province de Québec. Ici on pourrait bénéficier, tant au point de vue spirituel que temporel, de la présence des Trappistes. En effet, au cours de cette période, la province de Québec est aux prises avec les problèmes de l'émigration vers les Etats-Unis, de l'urgence du rapatriement et de l'essor à donner à la colonisation. Les Cantons de l'Est, parmi d'autres régions, manquent de Canadiens français cultivateurs et de spécialistes en agriculture. Ils auront profit à en trouver chez les Trappistes dont la réputation internationale en agriculture n'est plus à faire. D'autre part, nul n'ignore le bénéfice spirituel que retire une Église diocésaine de la présence de religieux contemplatifs.

Sous le titre N'émigrez pas, Le Pionnier du 8 avril 1881 dénonce, prenant à son compte les propos d'un prêtre du Nouveau-Brunswick, « l'erreur funeste dans laquelle tombent ceux qui croient améliorer leur position en allant user leur santé, leurs forces au profit des industriels yankees, qui en retour ne leur donnent que le moins de gages possible ${ }^{5}$. Le 25 février précédent, le même hebdomadaire écrit : "La Presse française de la Province de Québec, surtout la presse conservatrice, s'est élevée avec raison contre l'œuvre infâme des embaucheurs canadiens, qui viennent engager les familles de nos campagnes à prendre le chemin des Etats-Unis. Il nous faisait peine de voir de bonnes familles, vivant peut-être un peu à l'étroit, mais paisibles et tranquilles, aller manger le pain de 'exil $^{6}$. "

Le grand remède qu'on propose à l'émigration vers les ÉtatsUnis, c'est la colonisation, en particulier celle des Cantons de l'Est. La colonisation est aussi l'instrument par excellence du rapatriement. Mais, pour favoriser le rapatriement par la colonisation, il faut une organisation adéquate. À Sherbrooke, on s'évertue à y pourvoir. En effet, en quelques mois à peine, apparaissent tour à tour à Sherbrooke différentes institutions dont nous pouvons retracer les origines et percevoir les objectifs à travers l'hebdomadaire local.

5 Le Pionnier, Sherbrooke, 8 avril 1881.

6 Le Pionnier, Sherbrooke, 25 février 1881. 
En février 1881, le Pionnier annonçait l'établissement à Sherbrooke du Crédit Foncier. «Le but des promoteurs du Crédit Foncier étant de venir en aide à la classe agricole en réduisant le taux de l'intérêt qu'elle était forcée de payer jusqu'à présent,... ». «L'argent ne fera pas défaut, on en aura autant que l'on voudra, pourvu que les garanties soient suffisantes » et, ajoute-t-on, "Les Cantons de l'Est sont bien vus par les directeurs ». Le notaire Élisée Noël en est le représentant à Sherbrooke 7. Exactement un mois plus tard, jour pour jour, Le Pionnier toujours enthousiaste lorsqu'il s'agit de colonisation, annonce : « La Compagnie de Colonisation et de Crédit des Cantons de l'Est inaugure en ce moment ses opérations à Channay, dans le Canton de Woburn », et le rédacteur ajoute qu'il a appris, dans une entrevue avec M. J.-A. Chicoyne, un des promoteurs de l'entreprise, que « les amis sincères de la colonisation salueront avec bonheur cette partie du programme de la compagnie... 》 Enfin, on apprend que «cette belle association 》 «compte parmi ses actionnaires des membres influents et distingués du parti légitimiste en Bretagne et en Vendée 8 》. Le 24 mars suivant, on apprend que «La Société de Colonisation de la Cité de Sherbrooke (...) sous la présidence de sa grandeur $\mathrm{M}^{\mathrm{gr}}$ Racine, vient d'être officiellement reconnue par le gouvernement ${ }^{9}$ ". Le viceprésident est l'abbé Eugène Peigné, chanoine honoraire de Nantes. Parmi les directeurs, on trouve l'abbé H.-O. Chalifoux de Sherbrooke et M. J.-A. Chicoyne, avocat. Tous sont des "apôtres 》 de la colonisation. Le $1^{\text {er }}$ avril suivant, sous le titre Une excellente nouvelle, les lecteurs de l'hebdomadaire sherbrookois apprennent qu' «Une agence de Colonisation et d'Immigration vient d'être établie à Sherbrooke par notre gouvernement provincial ». Les Cantons de l'Est sont donc favorisés, mais ils ont besoin de toute cette aide qu'on leur apporte. «Cette région renferme encore plus de DEUX MILLIONS d'acres de forêt vierge qui n'attendent que la cognée et la pioche du défricheur pour se transformer en champs fertiles.» De plus, les Cantons de l'Est peuvent, mieux que d'autres régions, faciliter le rapatriement : «Placés dans le voisinage immédiat des EtatsUnis, ces cantons sont à la portée de nos compatriotes ${ }^{10}$, ceux qui se sont exilés.

7 Le Pionnier, Sherbrooke, 11 février 1881.

8 Ibidem, 11 mars 1881.

9 Le Pionnier, Sherbrooke, 24 mars 1881.

10 Le Pionnier, Sherbrooke, $1^{\text {er }}$ avril 1881. 
L'organisation ainsi mise sur pied pour lutter contre l'émigration des Canadiens français vers les États-Unis, pour favoriser le rapatriement de ceux qui y étaient déjà et pour promouvoir la colonisation dans les Cantons de l'Est, est si parfaite que certains « loyaux britanniques » de la région y voient, de la part de $\mathrm{M}^{\mathrm{gr}}$ Antoine Racine, évêque de Sherbrooke, et de Jérôme-Adolphe Chicoyne, agent de colonisation de la province de Québec, « un plan systématique pour l'éviction des anglo-protestants des Cantons de l'Est ${ }^{11}$ ». Mais d'autres, au contraire, y voient une protection pour le pays. Ainsi, J.-H. Pope, le député fédéral de Compton, lui-même "loyaliste», « un vrai tory », considère que "les fortes traditions canadiennesfrançaises offrent un obstacle à l'envahissement américain 12 ». Exprimant ce point de vue, Pope reprend une idée chère à Lord Elgin, exprimée une trentaine d'années plus tôt. Elgin souhaitait, en effet, «garnir la frontière de Français et les terres de l'intérieur de Britanniques qui pourront ainsi conserver l'amour de leur pays d'origine et de ses institutions à une distance suffisante des influences américaines ». Pour parvenir à cette fin, Lord Elgin ne voyait d'autre instrument que «le clergé, la plus puissante influence du BasCanada ${ }^{13}$ ». Quoi qu'il en soit des jugements portés antérieurement ou à l'époque même, les intentions de $\mathrm{M}^{\mathrm{gr}}$ Racine et de JérômeAdolphe Chicoyne sont infiniment plus pures que les sentiments que leur prêtent certains «loyaux britanniques» et J.-H. Pope a bien raison, pour sa part, de les appuyer.

Comme on l'aura remarqué, les amis de la colonisation dans les Cantons de l'Est - c'est ainsi qu'ils se désignent eux-mêmes et les opposants de la crise anti-catholique et laïcisante française se donnent la main. On va donc tenter d'établir, à la faveur de la crise qui sévit en France contre les congrégations, des Trappistes dans les Cantons de l'Est.

En 1880, Jérôme-Adolphe Chicoyne est de passage en France, à titre d'agent de colonisation. Il profite de l'occasion pour aller à l'abbaye de Melleray, saluer un de ses compatriotes Vertume Péloquin, en religion Frère Jérôme, prêtre Trappiste. Ce Canadien est entré dans l'ordre au Monastère du Saint-Esprit, premier établis-

11 Robert Rumilly, Histoire de la Province de Québec, vol. 4, p. 217.

12 lbidem, p. 218.

13 Cité par Mason WADE, Les Canadiens Français de 1760 d nos jours, traduit de l'anglais par Adrien VeNNE, Tome I : (1760-1914), Montréal, Le Cercle du Livre de France, 1963, p. 289. 
sement trappiste au Canada, à Sainte-Justine de Dorchester ${ }^{14}$. Cette fondation s'étant avérée insoutenable, le père Jérôme a fini par se retrouver à l'Abbaye de Melleray, en Bretagne. Vu les conditions en France à l'époque, les autorités de Melleray décident de permettre au Père Jérôme de passer au Canada avec Monsieur Chicoyne qui y retourne. Le Père Jérôme a pour mission de voir s'il ne se trouverait pas dans ce pays un endroit où établir la communauté au cas où les anticléricaux arriveraient à leur fin. Le 2 novembre 1880, Chicoyne et le Père Jérôme sont à La Patrie, dans le canton de Ditton. Aussitôt, il est question de l'établissement d'une Trappe à La Patrie, et Le Pionnier s'en réjouit en faisant valoir les avantages matériels de la présence des Trappistes : "Tout en condamnant la persécution qui détermine les Trappistes à jeter les yeux sur notre pays pour $y$ fonder des maisons de leur ordre, nous devons nous estimer heureux de les voir s'établir au milieu de nous. À ne les considérer qu'au point de vue des intérêts matériels seulement, ils sont appelés à nous rendre d'immenses services》. "Partout où leurs monastères existent, ils donnent l'exemple des améliorations agricoles et se placent à la tête de tous les progrès dont l'agriculture est susceptible. " Et encore : «On voit figurer leurs produits aux expositions, et les agriculteurs sont toujours cordialement admis à visiter les fermes de la Trappe, à y prendre des conseils et des renseignements ${ }^{15}$ ".

C'est dire en quelques lignes tout ce qu'on espérait des Trappistes pour le développement de l'agriculture dans les Cantons de l'Est. L'enthousiasme est à son comble, mais les Trappistes sont loin d'être établis de façon permanente à $L a$ Patrie. Le 8 avril 1881, Le Pionnier annonce : "Vendredi dernier, le premier avril courant, le Rév. P. Jérôme a célébré une première messe à la Trappe de Bethléem. Presque tous les colons de la paroisse y assistaient (...) Les assistants n'oublieront jamais le bonheur qu'ils ont eu d'assister à la naissance de cet établissement qui, avec le secours de la Providence, ne peut manquer de grandir et prospérer. Le nom de Bethléem est heureusement choisi : c'est d'un bon augure ${ }^{16}$. " Six mois plus tard, on se réjouit encore : «La Trappe de Bethléem, Canton de Ditton,

14 Sur cet essai de fondation, on lira avec profit un article d'André Côté, L'établissement des trappistes à Sainte-Justine de Dorchester, 18621872, dans Protée, décembre 1973, vol. 3, no 1, pp. 71-85.

15 Le Pionnier, Sherbrooke, 29 avril 1881.

16 Le Pionnier, Sherbrooke, 8 avril 1881. 
établie par la Grande Trappe de Melleraye, vient de recevoir, de la maison-mère, un bon renfort. Deux frères de ce séculaire couvent viennent d'arriver à Bethléem, au grand bonheur du Révd. Père Jérôme qui pourra maintenant jouir de la vie de communauté et de prières communes, après laquelle il soupirait depuis longtemps ${ }^{17}$. »

Un visiteur de Bethléem écrit en juin 1881: «Le nom de Bethléem réveille, chez tous les chrétiens, les sentiments de la plus vive reconnaissance (...) Il est un coin de terre dans les Cantons de l'Est qui est assez béni pour avoir mérité de porter ce nom. » Et notre visiteur continue: "C'est dans cet endroit, sur le bord d'un petit ruisseau, au milieu d'une forêt épaisse, qu'un humble religieux de Melleraye, en France, conduit par la main par la divine providence est venu établir son ermitage et fonder une de ces maisons de prières et de pénitence qui rappelle les temps des anciens anachorètes de la primitive Église. » Mais le visiteur passe rapidement aux intérêts temporels : "L'industrie agricole de la Province ne trouvera pas moins son compte dans l'établissement de cette Trappe (...) Les Trappistes sont essentiellement les cultivateurs modèles de l'univers entier, les plus courageux, les plus industrieux, les plus parfaits colons que la terre ait produits». Et plus loin : «Les habitants de la colonie semblent avoir reçu une nouvelle dose de courage en voyant arriver au milieu d'eux ces auxiliaires d'un nouveau genre. Si le scandale est pernicieux, le bon exemple a un effet tout opposé et il produit les meilleurs résultats. (...) Plus d'une localité enviera l'avantage incalculable dont La Patrie va être doté ${ }^{18}$. 》

La presse française légitimiste ne le cède pas en enthousiasme à la presse locale. Ainsi, L'Observateur de Nantes commente l'œuvre du Père Jérôme au Canada : "Après avoir visité et étudié, après avoir consulté la volonté divine et l'expérience des hommes, il choisit une vallée solitaire, au sein de la forêt vierge des Cantons de l'Est (...) il s'occupe depuis à jeter les bases d'un établissement appelé à rendre d'éminents services à cette région (...) Deux autres religieux de la Melleray sont allés le rejoindre dernièrement (...) L'établissement des Trappistes dans ces parages va donner une impulsion toute nouvelle et vigoureuse à leur développement. L'œuvre de la colonisation va décidément y entrer dans une ère de prospérité que tous les amis du bien salueront avec bonheur ${ }^{19}$."

17 Le Pionnier, Sherbrooke, 2 septembre 1881.

18 Le Pionnier, Sherbrooke, 3 juin 1881.

19 Reproduit dans Le Pionnier, Sherbrooke, 16 septembre 1881. 
Mais après l'arrivée de deux frères convers tout ne va pas aussi bien à Bethléem qu'on ne le croit dans le grand public. Le Père Eugène, abbé de Melleray, s'en inquiète. Le Père Jérôme est soupçonné d'irrégularité et la maison-mère demande à l'évêque de Sherbrooke de s'informer de sa conduite ${ }^{20}$. Mais, il n'y a pas lieu de s'inquiéter ${ }^{21}$. Les différends entre le Père et ses confrères peuvent s'expliquer par le petit nombre des religieux, la différence de mentalité et les besoins du milieu. Cependant, un Père, deux frères, une maison-chapelle, et quelques arpents de terre défrichés ne constituent pas une Trappe comme celle dont on fait l'éloge dans les journaux du temps, et le coût élevé de l'entreprise, ajouté aux difficultés entre les religieux, font que les autorités de Melleray doivent étudier la situation de plus près et prendre une décision définitive, car la fondation de Bethléem est beaucoup plus fragile qu'on veut bien le laisser penser.

L'essai de fondation trappiste à La Patrie, quoi qu'on en ait dit ou pensé, n'est rien d'autre qu'une tentative au sens le plus strict du terme, comme le font voir les documents. Le Frère Eugène, abbé de la Trappe de Notre-Dame de Melleray, écrit lui-même : «J'ai cru prudent (étant donné les circonstances) d'envoyer mon P. Jérôme avec Monsieur Chicoyne qui retournait au Canada, afin de voir si ce pays ne nous offrirait pas un asile plus sûr et plus tranquille que les diverses contrées de l'Europe ». L'abbé de Melleray précise la raison pour laquelle il a choisi le Père Jérôme : «Mon P. Jérôme comme étranger (il était Canadien) devait en cas d'expulsion être reconduit à la frontière; et je voulais lui éviter le désagrément en lui faisant prendre les devants ». Et l'abbé d'insister : «Mon but alors n'était que de prendre des renseignements précis sur la possibilité et l'avenir d'une trappe au Canada ». Dom Eugène précise: «Il pourrait bien se faire que nous n'allions pas au Canada de sitôt " et ajoute : «En tout cas si cette fondation ne réussit pas, nous y aurons risqué très peu d'argent. Et c'est pour ce motif que j’ai recommandé au Père Jérôme de ne point aller trop

20 F.M. Eugène, Abbé à Mgr Racine, Melleray, 9 décembre 1881, AAS, boîte St-Pierre de La Patrie, chemise 7.

21 Nous n'avons pas la lettre de Mgr Racine au F.M. Eugène, mais une lettre de ce dernier à l'évêque de Sherbrooke en date du 19 janvier 1882 montre que $\mathrm{Mgr}^{\mathrm{gr}}$ Racine était très satisfait de la conduite du F. Jérôme, AAS, boîte St-Pierre de La Patrie, chemise 7. 
vite, mais de se contenter pour le moment du nécessaire $22 \%$. Le mandat du P. Jérôme n'est donc pas aussi important que le croient ou veulent bien le laisser entendre les amis de la colonisation dans les Cantons de l'Est. En fait, l'œuvre, à peine née, touche déjà son déclin.

On a prétendu qu'en avril 1882, Dom Chouteau est délégué par l'abbaye de Melleray et que ce dernier a décidé de mettre un terme à la fondation du Père Jérôme à cause des difficultés de communication, de l'absence d'un chemin de fer, des longs mois d'hiver pendant lesquels les religieux étaient paralysés, immobilisés, ainsi que des lourdes dépenses qu'occasionnait l'entreprise ${ }^{23}$. La vérité toute nue est un peu différente. D'abord, c'est le P. Eugène, abbé de Melleray lui-même qui visite les lieux et prend la décision de mettre un terme à l'expérience de Bethléem. L'abbé de Melleray reprend par écrit dans une lettre à $M^{\text {gr }}$ Racine, en date du 29 août 1882 , les explications qu'il a déjà données de vive voix à l'évêque. Il parle de ses «impressions pénibles au sujet de cette entreprise. Je la trouvais au-dessus de nos forces, et de nos ressources ». L'abbé fait remarquer qu'il faudrait y envoyer des religieux en plus grand nombre et y investir un capital plus considérable. Or, en France, le personnel manque, les religieux de Melleray ne quittent pas la France, et les vocations se font rares. En conséquence, le Père Eugène se refuse à « laisser végéter cet essai de fondation dans l'isolement et la pauvreté ». Enfin, l'abbé fait allusion à la mésentente qui règne entre les religieux, le Père Jérôme et ses deux frères convers, il rappelle qu'il a ordonné aux deux frères de partir et ajoute : «si je ne veux pas perdre le $P$. Jérôme, je devrai le rappeler au plus tôt ». Dans cette même lettre, l'abbé informe l'évêque qu'il a chargé M. Chicoyne de louer ou de vendre l'établissement et que l'abbayemère s'attend à subir une perte sensible. Certes, le $P$. Eugène regrette le geste qu'il doit poser, mais il doit faire contre mauvaise fortune bon cour comme il l'explique à $M^{\text {gr }}$ Racine : "Que faire avec des éléments si peu religieux, qui ne sauraient vivre en paix », et plus loin : « $\mathrm{I}$ vaut mieux nous retirer au plus tôt ${ }^{24}$ ». Voilà, la décision est prise.

22 F.M. Eugène, Abbé à Mgr Racine, Melleray, 29 janvier 1881, AAS, boîte St-Pierre de La Patrie.

23 Albert Gravel, Les Cantons de l'Est, Sherbrooke, édité par l'auteur 1939, p. 195.

24 F.M. Eugène, abbé à $M^{g r}$ Racine, Melleray, 29 août 1882, AAS, boîte St-Pierre de La Patrie, chemise 7. 
L'évêque semble avoir pris la défense du P. Jérôme et le Père abbé reconnaît le mérite de son religieux, mais ajoute : "Il faut avouer qu'il lui manquait la plus élémentaire prudence en affaires (...) il est parvenu à élever le chiffre de nos dépenses à 40,000 francs au moins... ». Sur l'expression de la volonté de son supérieur, le Père Jérôme devra rentrer en France avec le R. Père Abbé de Bellefontaine qui passe au Canada et qui doit retourner en France en juin 1883. En attendant, le Père Jérôme vivra au presbytère d'Emberton «en vrai trappiste dans les exercices de la retraite et de la prière ». Son abbé le confie aux bons soins de $\mathrm{M}^{\mathrm{gr}}$ Racine : "Il est, Monseigneur, sous votre dépendance et il doit vous obéir ${ }^{25}$ ".

Les amis de la colonisation dans les Cantons de l'Est ont fondé beaucoup d'espoir sur le monastère de Bethléem. Au cours de l'été 1882, alors que la décision de mettre un terme à cette expérience est déjà prise, on parle toujours de Bethléem comme d'une maison destinée à prospérer et à servir de modèle aux cultivateurs. Le Pionnier du 10 août 1882 rapporte en effet : "Dimanche dernier plusieurs cultivateurs des environs sont venus visiter les travaux agricoles des RR. PP. Trappistes de Bethléem. Rien de plus beau à voir que ces champs cultivés dans le silence de l'oraison. Bien que le temps des semailles n'ait pas été favorable, la récolte sera bonne. Le blé a une apparence magnifique. Le R.P. Jérôme et ses confrères sont la joie et l'honneur de tous les cultivateurs de toute la contrée et nous ne cessons de remercier le ciel d'avoir placé ces bons religieux au milieu de nous ${ }^{26}$. Mais les amis de la colonisation devront se résigner à avoir vécu un rêve et il faudra attendre vingt ans exactement, soit de 1883 à 1903, avant que des Trappistes ne songent à s'établir dans les Cantons de l'Est.

Le conflit entre l'Église et l'État en France qui a commencé par la grande affaire de la politique scolaire finit par s'élargir et englobe bientôt toute la question des congrégations, enseignantes ou non. De 1901 jusqu'à la séparation de l'Église et de l'État en 1905, la situation s'aggrave et toutes les communautés, y compris celle des Trappistes, s'alarment avec raison. En 1899, une publication officielle a énuméré 3,216 congrégations existantes, dont la fortune serait

25 F.M. Eugène, abbé à Mgr Racine, Melleray, 4 janvier 1883, AAS, boîte St-Pierre de La Patrie, chemise 7.

26 Le Pionnier, Sherbrooke, 10 août 1882. 
évaluée à 1,071,775,260 francs. En conséquence, on parle du " milliard des congrégations ", milliard qui serait derrière les murs des couvents. En 1901, une loi, dite « loi sur les associations》, constitue un acte vigoureux de politique anticléricale, en s'attaquant à l'existence même des congrégations. Quatre-vingt-quatre communautés d'hommes et cent cinquante de femmes refusent de se soumettre à la loi. On transforme ainsi une loi de contrôle en une loi d'exclusion, et une autre loi de juillet 1904 retire toutes les autorisations accordées aux congrégations enseignantes ${ }^{27}$. En 1905, c'est la séparation de l'Église et de l'État. C'est dans ce contexte, pour le moins inquiétant, qu'une communauté de Trappistes songe sérieusement, semble-t-il, à s'établir dans les Cantons de l'Est, dans le diocèse de Sherbrooke.

En 1903, Dom Antoine, abbé d'Oka depuis 1892, est en Europe pour le Chapitre général. C'est de là qu'il demande au Père Adélard de solliciter de $\mathbf{M}^{\mathrm{gr}}$ Laroque, évêque de Sherbrooke, la permission d'établir une maison de l'ordre dans son diocèse. La démarche est aussitôt entreprise. « Le décret d'expulsion sera compulsoire à courte échéance et nos pauvres Religieux tendent leurs mains vers notre cher et hospitalier Canada », écrit le Père Adélard à $M^{\mathrm{gr}}$ Laroque, poursuivant : "Mon très Révérend Père Abbé (...) me prie de demander si Votre Grandeur serait disposée à permettre un établissement, probablement fixe, de Religieux ou Religieuses de notre Ordre dans son diocèse ». Le Père Adélard demande des renseignements sur la propriété que l'évêque de Sherbrooke aurait en vue, sur le coût probable de cette propriété, "sur la nature du sol de la région et sur les produits qu'on pourrait en tirer et sur la proximité des grandes voies de communications. L'affaire est urgente ${ }^{28}$ ».

C'est $\mathbf{M}^{\mathrm{gr}}$ Hubert Olivier Chalifoux, vicaire général, qui répond au nom de $\mathrm{M}^{\mathrm{gr}}$ Laroque. Aucune allusion à la permission épiscopale, cela va comme de soi. La région de Mégantic est toujours à l'honneur. $M^{\text {gr }}$ Chalifoux écrit en effet : " Dans le Canton de Louise, sur les limites du diocèse et tout près de la ligne provinciale séparant le Canada de l'Etat du Maine, il y a une ferme d'environ 100 acres en culture et adjoignant cette ferme des centaines d'acres de front

27 Maurice BAUMONT, L'essor industriel et limpérialisme colonial (1878-1904), Paris, PUF, 1949, Collection Peuples et civilisations, pp. 518-520.

$28 \mathrm{Fr}$ M. Adélard, secrétaire à Mgr Larocque, Oka, 29 septembre 1903, AAS, Carton Cisterciens-Trappistes (O.C.R.). 
à vendre ». $\AA$ toutes les autres questions, le vicaire général répond : «Je sais le sol très productif et d'une culture facile». Pour bref que soit le propos du vicaire général, il ne se montre pas moins hospitalier. $\mathrm{M}^{\mathrm{gr}}$ Chalifoux ajoute en effet: « $\mathrm{Si}$ quelques uns de vos Pères désirent venir voir les townships ils voudraient bien m'en informer puis je leur tracerai un programme qui leur sera d'un grand secours pour se renseigner ${ }^{29}$ 》.

Malgré les bonnes intentions de part et d'autre, le projet demeure sans suite. Dom Antoine en donne lui-même les raisons dans une lettre qu'il adresse à $\mathrm{M}^{\mathrm{gr}}$ Laroque en février 1904. Dom Eugène, Abbé de Notre-Dame, tente une fondation en Suisse. «Il ne peut donc pour le moment donner suite à son projet de fondation en Amérique, mais il n'y renonce pas ${ }^{30}$ ». Le projet de 1903 qui semblait si sérieux n'a pas de suite; du moins, on n'en trouve pas trace dans les archives archiépiscopales de Sherbrooke. D'ailleurs, les Trappistes ont été sauvés "à la suite d'une intervention directe de Dom Chautard, Abbé de Sept-Fons, auprès de Clémenceau ${ }^{31}$ », de sorte qu'une fondation dans les Cantons de l'Est devient inutile, pour le moment, du moins.

En effet, la séparation de l'Église et de l'État en France, réalisée en 1905, n'a pas donné entière satisfaction aux anticléricaux radicaux et Pointcaré écrit dans son journal «qu'un mois avant la guerre [la guerre de 1914] le Conseil des ministres était encore absorbé par la discussion des mesures à prendre pour en finir avec les congrégations ${ }^{32}$ ". D'où, en 1914, nouvelle demande des Trappistes pour s'établir dans le diocèse de Sherbrooke.

Cette fois, la demande vient par l'intermédiaire du Frère Dominique, supérieur du Monastère du Petit-Clairvaux, à Big-Tracadie en Nouvelle-Écosse. "Les nouvelles lois persécutrices que projette le gouvernement Français, écrit le Père Dominique, de plus en plus ennemi de la religion, obligeront sans doute dans un avenir prochain, nos frères de France à nous rejoindre ${ }^{33}$." Pourquoi recourir à

29 H.O. Chalifoux, vicaire général au R.F. Adélard, Sherbrooke, 5 octobre 1903, AAS, Carton Cisterciens-Trappistes (O.C.R.).

30 F.M. Antoine, abbé à Mgr Laroque, Oka, 22 février 1904, AAS, Carton Cisterciens-Trappistes (O.C.R.).

31 Latreille et autres, op. cit. p. 544.

32 Ibidem, p. 547.

33 F. Dominique, supérieur à l'évêque de Sherbrooke, Monastère du Petit-Clairvaux, Big-Tracadie, Nova-Scotia, 10 juin 1914, AAS, Carton Cisterciens-Trappistes (O.C.R.). 
l'évêque de Sherbrooke, alors que le Monastère du Petit-Clairvaux ne compte que 23 religieux? Ce monastère existe depuis dix ans et n'a pas trouvé une seule vocation en Nouvelle-Écosse. Pour cette raison, les moines de ce monastère devoient chercher ailleurs un milieu plus catholique. Ils le font pour eux-mêmes et aussi pour les religieux de l'abbaye-mère, Notre-Dame de Tymadeuc. Si la situation matérielle du Petit-Clairvaux à Big-Tracadie est excellente, l'absence de vocation, en milieu protestant, même sympathique, force donc les Trappistes à songer à d'autres lieux et c'est vers Sherbrooke qu'on regarde.

Le Père Dominique ne laisse planer aucun doute sur le problème que pose l'absence de vocation : «Votre Grandeur, écrit-il, pourraitelle nous offrir ou nous trouver dans son diocèse quelque chose qui nous convienne? en supposant que nous ayons des chances de nous recruter ». Le supérieur du Petit-Clairvaux insiste : «Votre Grandeur a déjà des représentants de plusieurs ordres religieux dans son diocèse; peut-être accueillerait-elle aussi favorablement une communauté de $\mathrm{La}$ Trappe et la pensée de nous trouver au milieu de Canadiens-Français, compatriotes et amis nous fait envisager à nousmêmes plusieurs chances de réussite dans cette partie de la province de Québec si vous voulez bien nous y admettre ${ }^{34}$.

Encore une fois, c'est le grand-vicaire Chalifoux qui répond au nom de $\mathbf{M}^{\mathrm{gr}}$ Laroque, de qui il deviendra l'évêque auxiliaire quelques mois plus tard [décembre 1914]. «Sa Grandeur me charge de vous informer, écrit le grand-vicaire, qu'au sud-est du diocèse de Sherbrooke, près de l'État du Maine, est le Lac Mégantic, long de douze milles et environné de terres à la culture (...) Si vous désirez visiter ces terrains, vous voudrez bien m'en informer afin que Messieurs les Curés des diverses paroisses vous reçoivent et vous fassent voir le pays. \$ La réponse est courtoise, mais sans trop d'enthousiasme : la permission reste à obtenir. Le vicaire général ajoute : «Cette visite terminée, vous vous rendrez à Sherbrooke afin de faire le rapport de votre voyage. Je prendrai note de vos observations et de vos projets, puis je communiquerai le tout à Sa Grandeur Monseigneur l'évêque, à son retour de Rome qui devra s'effectuer vers le commencement de novembre prochain ${ }^{35}$ ".

34 Ibidem.

35 H.O. Chalifoux, V.G. au Rév. Dominique, Sherbrooke, le 23 juin 1914, AAS, Carton Cisterciens-Trappistes (O.C.R.). 
Quelques jours plus tard, le 26 juin, le Père Dominique répond à $\mathbf{M}^{\mathrm{gr}}$ Chalifoux. Il semble beaucoup moins enthousiaste qu'au début du même mois, alors qu'il s'adressait à $\mathrm{M}^{\mathrm{gr}}$ Laroque. Il doit écrire à son Père Abbé, en France, avant de visiter la région du Lac Mégantic et il se demande \& quel parti on pourrait en tirer si le gouvernement de la province ou une âme charitable daignait nous $y$ offrir une concession ${ }^{36}$ 》.

La demande de 1914, comme celle de 1903, n'aura pas de suite. Ainsi, en trente-quatre ans, les Trappistes ont tenté de s'établir dans le diocèse de Sherbrooke et sollicité deux fois la permission de l'évêque de tenter de nouveau l'expérience. À chaque fois, l'autorité épiscopale s'est montrée sympathique. Mais il n'y a toujours pas de Trappistes dans les Cantons de l'Est dans les années 1940, alors que c'est l'évêque lui-même qui va prendre l'initiative de les y inviter. En 1944, en effet, $\mathrm{M}^{\mathrm{gr}}$ Desranleau demande à Dom Pacôme, abbé d'Oka, d'établir de ses religieux dans son diocèse. L'abbé d'Oka lui répond : «La guerre a grandement enrayé notre recrutement et il nous faudra attendre des temps meilleurs pour songer sincèrement aux fondations ${ }^{37}$ ». Mais l'évêque tient bon - c'est l'une de ses caractéristiques dominantes - et, en août 1948, il revient à la charge et fait une proposition ferme à Dom Pacôme. «Il me paraît que l'heure est venue de préparer un haut lieu de prières dans le diocèse; une trappe est ce qui serait de mieux, écrit l'évêque de Sherbrooke. Dites-moi oui et venez voir sans retard. $\mathbf{M}^{\mathrm{gr}}$ Desranleau a tout prévu. « $\AA 15$ milles de Sherbrooke, à 2 milles de la gare, en belle campagne, il y a une ferme d'anglais non-catholique, de plus de 400 arpents (...) à vendre pour environ $\$ 20,000$.; le bois seul, m'affirmet-on paierait le tout. Et il fait appel à la générosité de l'abbé d'Oka. « Il y a, me dit-on, plus de 75 Pères dans votre monastère, pourquoi ne pas m'en donner une demi-douzaine ? ${ }^{38}$. \$ C'est bien là le style déterminé de $\mathbf{M}^{\mathrm{gr}}$ Desranleau. Mais son ami Dom Pacôme sait y résister. \& Je suis confus de la confiance que vous mettez en nous et je vous remercie », écrit-il. Mais il ajoute aussitôt : "Malgré le désir que nous aurions d'accéder à votre demande, nous sommes forcés d'admettre que nous ne sommes pas encore prêts (...) il nous

36 F. Dominique à Mgr Chalifoux, Big-Tracadie, N.S., 26 juin 1914, AAS, Carton Cisterciens-Trappistes (O.C.R.).

37 F.M. Pacôme, Abbé à Mgr P. Desranleau, Oka, le 26 mai 1944, AAS, Carton Cisterciens-Trappistes [O.C.R.].

38 Mgr Desranleau à Dom Pacôme, abbé d'Oka, Sherbrooke, 20 août 1948, AAS, Carton Cisterciens-Trappistes [O.C.R.]. 
faut attendre que le recrutement des autres maisons leur permette de se soutenir sans la maison-mère ${ }^{39}$.

L'Église de Sherbrooke ne semble pas destinée à accueillir des Trappistes, mais elle doit fournir des sujets à divers monastères de cet ordre, même au-delà des frontières canadiennes. En effet, le carton Trappistes-Cisterciens, [O.C.R.] conservé aux archives de l'Archevêché de Sherbrooke montre que le diocèse - tenant compte du caractère toujours exceptionnel de la vocation monastique - a fourni bien des fils à l'ordre de saint Bernard.

À l'époque de la tentative de Bethléem, à La Patrie, dans le Canton de Ditton, on travaille ferme au progrès de la colonisation dans les Cantons de l'Est. Toute l'organisation mise sur pied à cette fin en témoigne. Les amis de la colonisation dans nos Cantons et des membres importants du parti légitimiste en France se donnent alors la main. Le rôle du chanoine Peigné, par exemple, n'est pas de mince importance. Et c'est grâce à certains légitimistes, amis de Jérôme-Adolphe Chicoyne, si les capitaux français jouent un rôle ici. Le fait que le Père Jérôme célèbre la première messe à Channay, entreprise nantaise, plus tard soutenue par le Crédit Foncier de Paris, n'est pas sans signification. Les Trappistes sont amis des légitimistes, semble-t-il. Si les Cantons de l'Est peuvent être considérés comme un asile possible pour les Trappistes de Melleray, ces derniers, en retour, semblent pouvoir servir les intérêts de la colonisation, nous prétendons l'avoir montré. Toute la publicité faite autour de la tentative de Bethléem met l'accent sur l'apport matériel que peut constituer une maison de Trappistes. Ces Cisterciens de la stricte observance sont alors plutôt considérés comme des maitres d'agriculture que comme des maîtres spirituels.

Il en va tout autrement des demandes que font certaines maisons de Trappistes en 1903 et en 1914, en vue de s'établir dans les limites du diocèse de Sherbrooke. Si les circonstances sont alors aussi pénibles pour ces religieux en France, au Canada, dans les Cantons de l'Est en particulier, la situation n'est plus la même après vingt ans. La tentative de Bethléem a été une affaire publique autour de laquelle on a fait beaucoup de publicité. La demande de 1903, pour sa part, se résume à un échange de lettres entre l'Abbaye d'Oka et l'évêché de Sherbrooke. En 1903, certes il existe encore des pro-

39 Fr. M. Pacôme, Abbé à Mgr P. Desranleau, Oka, 24 août 1948, AAS, Carton Cisterciens-Trappistes [O.C.R.]. 
blèmes de colonisations dans la province de Québec, mais les Cantons de l'Est ne sont plus au premier rang. De plus, les affaires de la colonisation posent de tels problèmes que le gouvernement doit faire enquête. Et en 1905 - année de la séparation de l'Église et de l'État en France - le gouvernement provincial organise le «congrès de la colonisation pour clore l'agitation ouverte par le congrès de $1898{ }^{40}$ ». En 1914, la demande faite par le Père Dominique, supérieur du Petit-Clairveaux, en Nouvelle-Écosse, au nom de l'Abbaye de Notre-Dame de Tymadeux, est, elle aussi, simplement un échange de lettres entre ce religieux et le vicaire général du diocèse de Sherbrooke. Mercier a beau considérer que l'œuvre colonisatrice renferme « l'avenir et la prospérité de la province de Québec comme le salut de la race canadienne-française ${ }^{41}$, , le grand problème, en 1914, est celui de la guerre, et non pas celui de la colonisation. Quoi qu'il en soit, tant en 1903 qu'en 1914, ce sont les Trappistes eux-mêmes qui ont rebroussé chemin et non l'Église de Sherbrooke qui leur a fermé ses portes.

En 1944 et en 1948, alors que $\mathrm{M}^{\mathrm{gr}}$ Desranleau fait des démarches auprès de l'Abbaye d'Oka pour obtenir des Trappistes dans son diocèse, les circonstances ne sont pas favorables. La guerre, toujours la guerre.

Et toute cette démarche historique pour dire que le diocèse de Sherbrooke n'eut jamais d'établissement trappiste permanent. Mais il peut se consoler. Faute de «moines blancs», il a des «moines noirs ». En effet, depuis décembre 1912, les Bénédictins tiennent bon sur les bords du lac Memphrémagog. C'est là que l'Église de Sherbrooke a ce «haut lieu de prières » dont parlait $\mathrm{M}^{\mathrm{gr}}$ Desranleau, alors qu'il souhaitait ardemment la venue des Trappistes dans son diocèse.

Jean-Guy LAVALLÉE, Département d'Histoire, Université de Sherbrooke. p. 88.

40 Robert Rumilly, Histoire de la Province de Québec, vol. XII, 41 Ibidem, vol. XIX, p. 162. 